\title{
Comparación de los límites administrativos con los perceptivos del Barrio Evita, Ciudad de Río Gallegos
}

\section{Comparison of the administrative limits with the perceptives of the Evita District, City of Río Gallegos}

\author{
Gabriela A. Vidal, ggabvidal7@gmail.com \\ Cristian Ampuero, campuero@uarg.unpa.edu.ar \\ Alicia P. Cáceres, acaceres@uarg.unpa.edu.ar \\ Grupo de Investigación y Extensión Z. Terra Cognita \\ Instituto de Ciencias del Ambiente, Sustentabilidad y Recursos Naturales (ICASUR) \\ Universidad Nacional de la Patagonia Austral - Unidad Académica Río Gallegos \\ Av. Gregores y Piloto "Lero" Rivera S/N \\ Río Gallegos, Santa Cruz, Argentina
}

Recibido: 03/05/18. Aceptado: 29/08/18

\section{RESUMEN}

El problema de investigación se origina porque la Municipalidad de Río Gallegos ha delimitado el uso del suelo residencial en barrios sin considerar el proceso histórico de ocupación y la identidad cultural del mismo, sólo por cercanía. Este tipo de políticas municipales impacta en la identidad del barrio. En el caso del Evita, al barrio histórico, claramente perceptible en la nueva delimitación, se han incorporado otros barrios, algunos construidos por sindicatos, otros por la entrega del municipio de tierras para el uso residencial, otros por la venta de lotes urbanos producto de la subdivisión de chacras improductivas. A los efectos de conocer cuáles son los límites del barrio Evita para el vecino, este trabajo tiene como objetivo estudiar si la delimitación administrativa del barrio Evita de Río Gallegos coincide con los límites perceptivos que los habitantes poseen de su propio barrio. Para abordar esta problemática se utilizó teórica y metodológicamente el enfoque de la geografía de la percepción y cuyos resultados servirán de aporte para una mejor gestión del barrio, como espacio vivido.

Palabras clave: Geografía de la percepción; Barrio Evita, Delimitación geográfica; Río Gallegos

\begin{abstract}
The research problem originates because the Municipality of Río Gallegos has delimited the use of residential land in neighborhoods without considering the historical process of occupation and its cultural identity, only by proximity. This type of municipal policies impacts the identity of the neighborhood. In the case of Evita, the historic district, clearly noticeable in the new delimitation, has incorporated other neighborhoods, some built by unions, others by the delivery of the municipality of land for residential use, others by the sale of urban lots product of the subdivision of unproductive farms. In order to know what the limits of the Evita neighborhood are for the neighbor, this work aims to study whether the administrative delimitation of the Río Gallegos neighborhood of Evita coincides with the
\end{abstract}


perceptive limits that the inhabitants have of their own neighborhood. To approach this problem, the geography of perception approach was used theoretically and methodologically, and its results will serve as a contribution to a better management of the neighborhood as a lived space.

Key words: Geography of perception; Evita neighborhood, geographic delineation; Río Gallegos.

\section{INTRODUCCIÓN}

A fines de la década de 1990, la Municipalidad de Río Gallegos delimitó administrativamente a través de la Ordenanza $\mathrm{N}^{\circ} 1686$ en 23 barrios el uso del suelo residencial de la ciudad, sin contemplar para ello el proceso histórico de ocupación ni la identidad de los habitantes en relación a su barrio. Esta política no fue acompañada de la consulta a los vecinos sobre qué opinaban ni tampoco informarles de los cambios.

Según esta Ordenanza, el Barrio Evita pertenece a la Circunscripción III, Sección E, y sus límites son: Noreste calle Zapiola, Este y Sur Av. Balbín (ex vías del ramal ferro industrial Río Turbio), Oeste calle Juan Manuel de Rosas.

El barrio histórico surge a principios de la década de 1990 ante la necesidad de evacuar y erradicar a las familias asentadas en terrenos ubicados en la zona del basural, terrenos fiscales, privados y estatales (YCF). La Municipalidad habilitó 2/3 manzanas en el sector denominado "Trencito Anexo", que benefició a 23 familias aproximadamente.

Debido a que la demanda de terrenos fiscales continúa y aumenta, el Municipio habilitó 6/7 manzanas más para lograr satisfacer las demandas de familias que no contaban con su terreno, pero sí poseían su vivienda ubicadas en barrios de inmigración chilena. Por este motivo, comienzan a llegar al barrio viviendas de material ligero transportadas sobre trineos de hierro, máquinas y camiones facilitados por el municipio.

Actualmente al barrio histórico se le han adosado sectores residenciales cercanos, conformando áreas homogéneas que poseen características constructivas y socioeconómicas diferentes entre sí.

Por este motivo, este trabajo tiene como objetivo estudiar la delimitación perceptiva de los habitantes del barrio Evita de la ciudad de Río Gallegos, para comparar si coincide o no con la delimitación administrativa realizada por el municipio.

Respecto al estado del arte de la temática, es importante destacar, que existe un amplio desarrollo del estudio de la Geografía de la Percepción en la ciudad de Río Gallegos y otros espacios de la Provincia, pero no en específicamente en el área de estudio seleccionada.

Este trabajo forma parte del informe final de la Beca Estímulo a las Vocaciones Científicas (EVC) del Consejo Interuniversitario Nacional (CIN), desarrollada por Gabriela Vidal durante el año 2017, dirigida por Alicia P. Cáceres y codirigida por Cristian Ampuero, en el marco del Proyecto de Investigación de Desarrollo Tecnológico y Social (PDTS) 29/A380 "Tercera franja residencial de Río Gallegos: diagnóstico territorial de áreas con ausencia y presencia de cobertura de servicios. Propuesta de localización de nodos de servicios", radicado en la Universidad Nacional de la Patagonia Austral, Unidad Académica Río Gallegos. 


\section{MARCO DE REFERENCIA}

\subsection{Marco histórico - geográfico}

Administrativamente Santa Cruz se divide en siete departamentos, el más austral es Güer Aike. La ciudad de Río Gallegos es la cabecera departamental y capital provincial. Según los resultados definitivos del Censo Nacional de Hogares, Población y Vivienda del año 2010, su población total es de 95.796 hab, en donde habita el 85\% de la población departamental (INDEC, 2012). (Fig. 1)

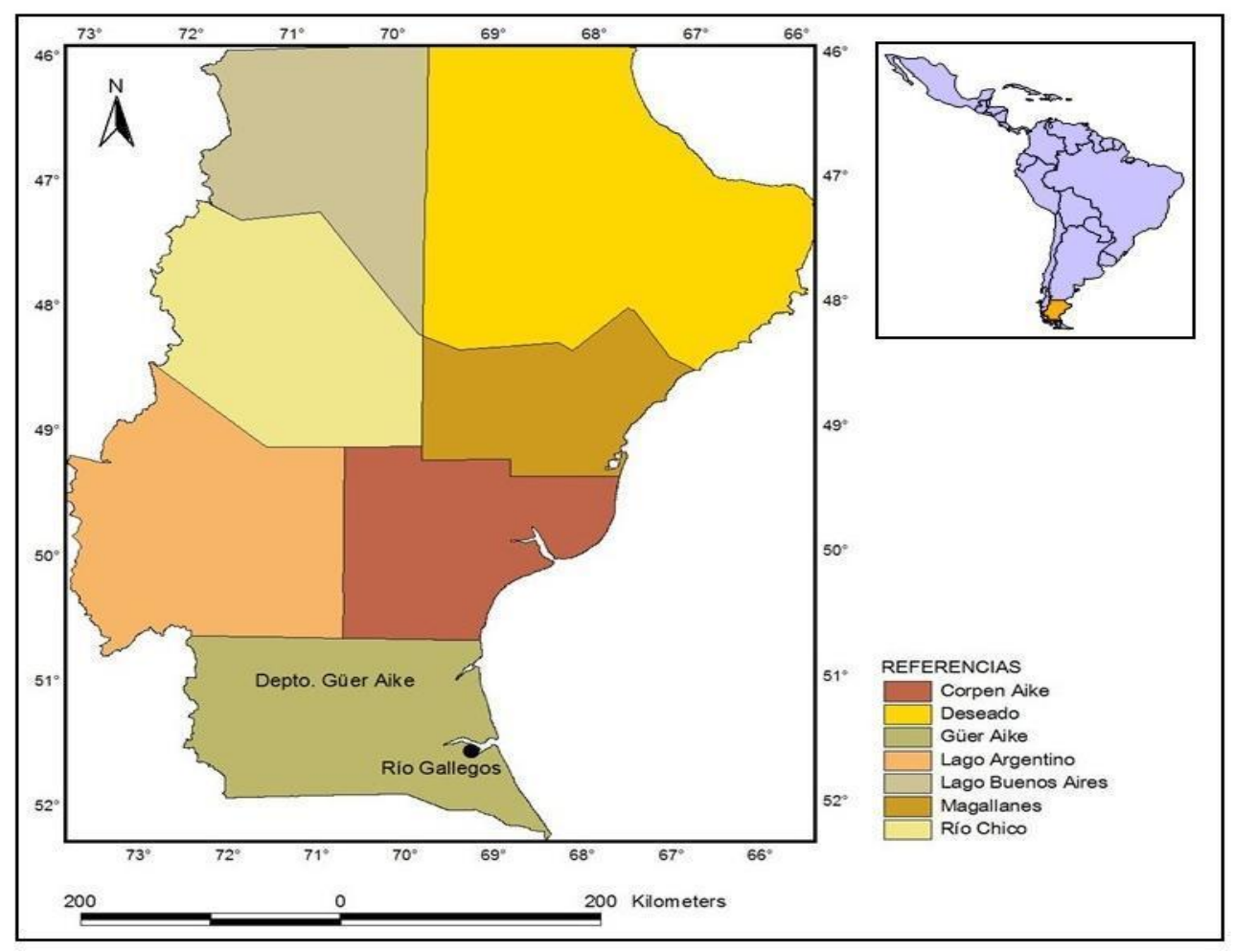

Fig. 1: Provincia de Santa Cruz, República Argentina Fuente: Elaboración propia.

El sitio del barrio Evita es $51^{\circ} 36^{\prime} 43,80^{\prime \prime}$ Lat. Sur 69 $14^{\prime} 50,91^{\prime \prime}$ Long. Oeste. La situación del barrio con respecto al resto de la cuidad es que se encuentra, aproximadamente a $2 \mathrm{~km}$ del centro de la ciudad de Río Gallegos. Su área ocupa parte del sector Noroeste y del sector Occidental de la localidad. El barrio tiene forma triangular y sus calles tienen un trazado algo irregular, limitan con los barrios Náutico, Nuestra Señora del Carmen y Yacimientos Carboníferos Fiscales. (Fig. 2) 


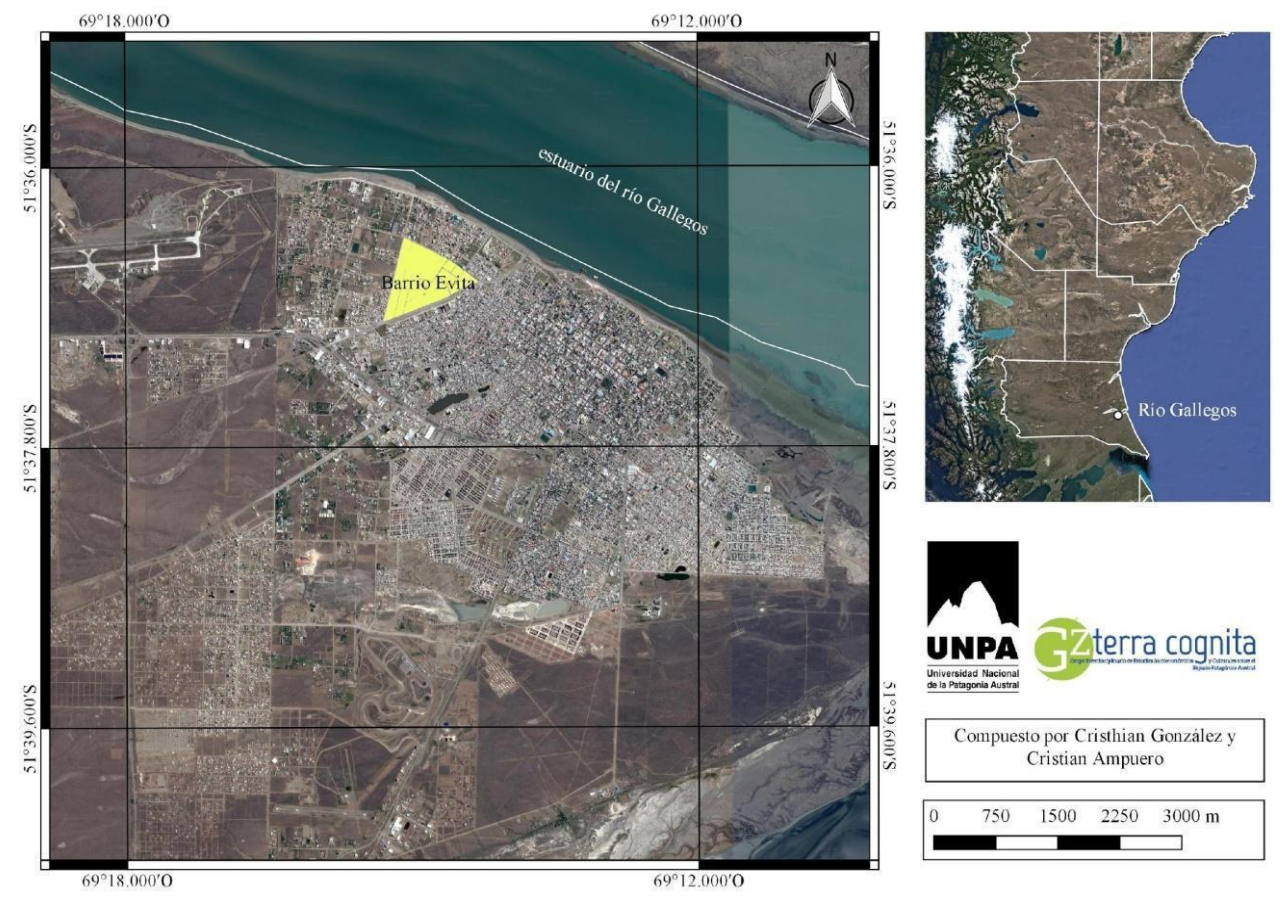

Fig. 2. Ubicación del barrio Evita en la ciudad de Río Gallegos

Fuente: Elaboración propia en base a información de la Municipalidad de Río Gallegos y tomando como base imagen satelital de Google Earth. Fecha: febrero de 2018.

Los límites del barrio están constituidos por las calles Zapiola al Norte, Av. Balbín al Este y Juan Manuel de Rosas al Oeste.

El primer Barrio se localizaba ubicado entre las vías, Pablo Neruda, Hijas de María Auxiliadora y Juan Manuel de Rosas. En el año 2000, se amplía hasta la calle Zapiola y Juan Manuel de Rosas y comprende Barrios como el Vial.

En el año 1980 con la conformación de la junta vecinal "El Trencito", se lleva a cabo el otorgamiento de un terreno fiscal con la intención inicial de la construcción de 50 viviendas para los habitantes del barrio.

En sus comienzos estaba dividido en dos sectores denominados "Trencito" y "Perejil. Las vías del ferrocarril estaban instaladas en donde hoy se encuentra la actual Av. Ricardo Balbín.

En esa época este barrio, solo era habitado por chilenos que arrendaban las tierras y construían sus casas precarias de material ligero, las cuales más tarde eran transportadas en trineos a terrenos de su propiedad, aunque muchas veces lograron adquirir a través de la municipalidad los terrenos para luego ser propietarios de los mismos.

La mayoría de las calles eran de ripio en las cuales se formaban enormes lagunas que tornaban el lugar intransitable y la iluminación era precaria y escasa.

La denominación del barrio como "Evita" data aproximadamente del año 2000, fecha que a su vez fue delimitado por la oficina catastral de la Municipalidad de Río Gallegos, que fija la jurisdicción que comprende en la actualidad. Es importante destacar que el ramal ferroviario, en su tramo entre la Av. Almirante Brown y la Rutas Nacionales $\mathrm{N}^{\circ} 3$ y $\mathrm{N}^{\circ} 40$, fue reemplazado por la Av. Ricardo Balbín, inaugurada en el año 2006, la cual permitió la continuidad en la red urbana entre el Área Central con la Segunda Franja Residencial y el Borde Urbano, hoy Tercera Franja Residencial (TFR) (Cáceres et al; 2013:56). El reemplazo del Borde Urbano por la Tercera Franja Residencial, resulta de "la demanda de viviendas, por un lado, presionó para que, desde el Estado provincial, se adoptara la decisión política de recuperar tierras fiscales ... aprovechar la oportunidad del cierre del Ramal Ferro industrial 
Río Turbio y su terminal en el Muelle homónimo, para permitir la continuidad en la red vial de vinculación del Área Central hacia el Oeste del ejido.

Del análisis de las transformaciones en el Borde Urbano como producto del avance urbano sobre barreras antrópicas, se observa como consecuencia la consolidación del área en la TFR de Río Gallegos, espontáneamente, sin planificación.

Si bien, ha resuelto parte de la demanda habitacional, el Estado municipal no reconoce, desde la gestión a la tercera franja residencial en su conjunto, hay ausencia de políticas de planificación urbana de modo integrado entre el Estado nacional, provincial y municipal. De este modo, se profundiza el carácter monocéntrico de Río Gallegos, la periferia no ofrece equipamiento, infraestructura, servicios que ofrece el centro: educación superior, educación especial, espacios cerrados privados para la práctica de deportes y recreación, comercios, salud, etc". (Cáceres et al; 2013:58)

\subsection{Marco teórico}

Entre 1989 y 1991 se realizó la primera investigación sobre geografía de la percepción en Santa Cruz, ya que no había antecedentes de estudios geográficos con este enfoque en ningún asentamiento urbano del territorio santacruceño. Alicia P. Cáceres con Ester Visciglia y Miriam Vázquez bajo la dirección de Mariano Zamorano llevan a cabo el primer estudio sobre geografía de la percepción en la provincia de Santa Cruz, concretamente en la ciudad de Río Gallegos. ${ }^{1}$

La geografía de la percepción, es una corriente del pensamiento geográfico que aborda el aspecto metodológico como así también el teórico. Vilá Valentí (1983) la define “como un enfoque geográfico que entiende el espacio... en función de su valor subjetivo, como espacio conocido aprehendido individualmente; es el espacio vivido". 2

Frias (2011) cita a Capel (1973) "sostiene que este enfoque permite comprender las significaciones simbólicas que las personas construyen del espacio geográfico más próximo, las cuales inciden en su accionar con respecto al mismo. Es por ello que la geografía actual ha establecido "... el papel decisivo de la percepción humana en la formación de una imagen del medio real, la cual, y no éste, es la que influye directamente sobre su comportamiento".

Frias (2011) cita a Zamorano (1991) quien dice: el barrio está “... compuesto de islotes y calles, en un conjunto que constituye un medio de vida y de actividades dotado de individualidad... sectores homogéneos, espontánea o planificadamente, cuya superficie tiene un destino específico: residencia. Las formas variables, las delimitaciones indecisas, las interpretaciones frecuentes, los diseños alternativamente lineales o espaciales, no anulan la tendencia al agrupamiento, de acuerdo con el tipo de relaciones. Asimismo, cuando la superficie correspondiente es circunscrita como espacio administrativo, de gestión, suele suceder que no existe coincidencia con el barrio, en cuanto éste implica una vivencia especial para los ciudadanos".

Este autor sostiene que el barrio vivido por los vecinos en la actualidad presenta como problemática la pérdida de vivencias del espacio inmediato, es decir el barrio residencial, “...el habitante se siente sin influencias en la elaboración de su hábitat, y su incorporación a los barrios adocenados que le brindan los organismos públicos, sin una intervención activa de

\footnotetext{
1 AMPUERO, C. (2013). Percepción urbana de la localidad de El Calafate. Tesis de Licenciatura en Geografía. UNPA - UARG. Río Gallegos. Inédita. Pág. 38.

2 Citado por AMPUERO, C. (2013). Percepción urbana de la localidad de El Calafate. Tesis de Licenciatura en Geografía, UNPA - UARG. Inédito. Pág. 26.
} 
su parte, lo predisponen mal desde el punto de vista del ambiente propio, acerca del cual se ha perdido el sentimiento de arraigo y la ligazón, normalmente está signada por la provisoriedad". En función del marco teórico expuesto, se considera "barrio" a un conjunto de manzanas de uso residencial con identidad propia, dada esta identidad por la percepción de los vecinos como espacio vivido."

Respecto a la definición de barrio Triviño, Cáceres y Ampuero (2017) ${ }^{4}$ citan diversos autores provenientes de diversas disciplinas científicas. Desde la geografía, Zamorano (1985) los ha caracterizado como "...secciones de la ciudad que representan cierta identidad o carácter común, generalmente dado por el uso residencial, industrial, financiero, etc." (Zamorano, 1985:136). De esta forma establece que toda la ciudad queda dividida en "sectores homogéneos, espontánea o planificadamente, cuya superficie tiene un destino específico: negocios, industria, administración, residencia..." (Zamorano, 1992:75).

Desde la arquitectura, Aronovici (1965) considera que el barrio es el lugar físico o estructura urbana donde se asienta la comunidad vecinal, caracterizándolo como un sector delimitado y diferenciado, definido por ciertas características (por ejemplo, los contactos entre los individuos y los grupos menores que lo integran).

Ander-Egg (1995), desde la sociología considera que es una zona o espacio de la ciudad que posee límites físicos y simbólicos (que no coincide necesariamente con la división administrativa de la ciudad). En esta misma línea de análisis, su colega Gabriel Alomar (1980) evidencia a la "vecindad" o comunidad vecinal como unidad social habitante del barrio; la misma posee personalidad propia y conforma lo que podríamos denominar una "familia de familias", cuya principal característica es que los contactos entre los individuos y grupos que la integran son más o menos frecuentes y personales (además concluye afirmando que barrio es sinónimo de unidad vecinal).

La perspectiva antropológica de Gravano (1991), incorpora la identidad barrial como elemento que define al barrio y que trasciende lo puramente urbano, arquitectónico o espacial, presente en la mayor parte de las definiciones del concepto. El autor lo considera como "...un lugar común en la ideología de los habitantes de la ciudad, pues tiene una determinada eficacia para referir de un modo sintético a diversos aspectos de la realidad. Su sola mención encierra todo un mundo de significaciones". (Gravano, 1991:66)

Según Lynch (1960), son sectores de la ciudad que resultan inmediatamente identificables para los habitantes y usualmente reciben nombres locales. Este autor desde el enfoque de la percepción considera que el barrio puede ser abordado a través de elementos que identifican al mismo. Esta visión no es exclusiva de quienes residen en él, sino que personas ajenas a sector pueden igualmente percibirlo como un sector de la ciudad con identidad propia."

Siguiendo los lineamientos de Frias (2008) “...el barrio es un espacio geográfico que tiene mucho significado individual y como parte de un conjunto de personas que se identifican con él". 5 Por otro lado, Lynch (1984) plantea que "... su más destacada particularidad es la idea de pertenencia que provoca en sus moradores", siguiendo la afirmación de la imagen mental que se produce, el mismo autor define: "la representación que cada persona posee de su entorno, es un conjunto de elementos físicos y tangibles y otros aspectos, como son la significación de la zona, su funcionalidad, su historia o, incluso su nombre".

\footnotetext{
${ }^{3}$ ZAMORANO (1991) citado por FRIAS, P. (2008). La percepción de un barrio de la segunda franja residencial de Río Gallegos al antiguo barrio Belgrano. Pág. 2 y 3.

${ }^{4}$ TRIVIÑO, J. G.; CÁCERES, A. P.; AMPUERO, C. (2017). Delimitación perceptiva de un sector residencial de la ciudad de Río Gallegos. Informe final de Beca Estimulo a las Vocaciones Científicas CIN - UNPA. Río Gallegos. Págs. 6 y 7.

${ }^{5}$ FRIAS, P. (2008): La percepción de un barrio de la segunda franja residencial de Río Gallegos al antiguo barrio Belgrano. UNPA. Río Gallegos. Pág. 2.
} 
En coincidencia con lo que plantea Cáceres (2009) se puede concluir que el barrio se encuentra definido, por un lado, por la identidad de sus habitantes y, por otro lado, por su forma y función. La forma está relacionada con lo visible del sector, es decir, con los elementos que lo distinguen del resto y que no necesariamente son de tipo arquitectónico. La función está basada en el tipo de uso que se haga del suelo."6

\section{MATERIALES Y MÉTODOS}

Se emplearon tres tipos de técnicas para realizar el trabajo de investigación: el relevamiento bibliográfico, la observación directa y la encuesta.

En primer lugar, se realizó un relevamiento bibliográfico, tanto en bibliotecas reales como virtuales.

Se adoptó teórica y metodológicamente, la Geografía de la Percepción y concepto de barrio en virtud de entender al espacio, en este caso el Barrio Evita.

Se realizó una salida de campo el día 12 de mayo del 2017, con el objetivo de reconocer el área de estudio y recorrer los límites del barrio Evita establecidos por la Municipalidad de Río Gallegos.

En esta etapa de observación directa acompañado con mapas, imágenes satelitales y GPS, se descartaron otros usos del suelo que no fueran residencial, para determinar conglomerados residenciales, relevamiento que se completó en gabinete con imágenes satelitales históricas. Asimismo, se realizó un registró mediante un conteo cada parcela de los conglomerados para luego tomar de cada uno de ellos una muestra significativa y representativa territorialmente que permitiera aplicar un cuestionario de encuestas. Se determinaron 9 conglomerados (Fig. 3), para los cuales se estableció el tamaño de la muestra con la cantidad de encuestas a realizar en cada uno de ellos. (Tabla 1)

Posteriormente se diseñó el cuestionario de encuesta (Anexo 1) conformado por preguntas abiertas y cerradas, lo que facilitó que pudieran realizarse de forma rápida en cada vivienda. Cabe destacar que la selección de las viviendas se realizó a través de una técnica de muestreo bietápico, donde se seleccionó la vivienda a encuestar por conglomerado, el que estaba dividido en parcelas numerado desde 1 hasta el total de lotes residenciales sucesivamente siguiendo el sentido de las agujas del reloj.

Una vez aplicada la encuesta a la población, se diseñó una base de datos en planilla de cálculo (Microsoft Excel) donde en las filas se ubican las unidades de análisis con el identificador (ID) y en las columnas las respuestas obtenidas. Se cargaron las respuestas, las que posteriormente fueron codificadas, para recibir tratamiento estadístico, con el Software Statistical Package for the Social Sciences (SPSS). Se analizaron todas las respuestas a los efectos de obtener frecuencias absolutas y relativas, tanto de los datos censales, como los referidos a la percepción. Los datos tabulados fueron representados gráficamente con el fin de analizar la delimitación perceptiva que lo vecinos poseen del barrio Evita, que luego fue comparada con la delimitación administrativa generada por el municipio local.

\footnotetext{
6 TRIVIÑO, J. G.; CÁCERES, A. P.; AMPUERO, C. (2017). Delimitación perceptiva de un sector residencial de la ciudad de Río Gallegos. UNPA. Río Gallegos. Págs. 6 y 7.
} 


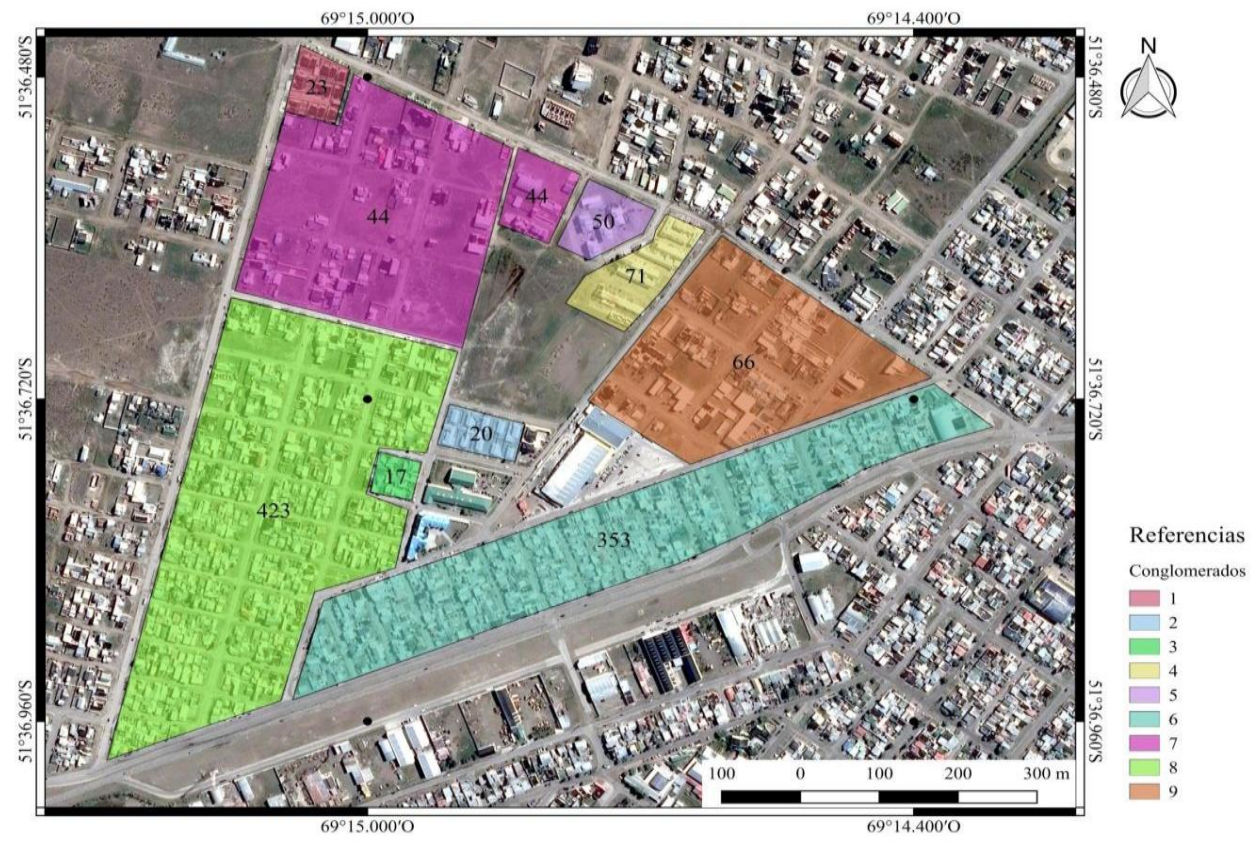

Fig. 3. Conglomerados definidos en el barrio Evita para el muestreo estadístico Fuente: Elaboración propia. Fecha: febrero de 2018.

\begin{tabular}{ccc}
\hline Viviendas por conglomerado & Tamaño 5\% muestra & Tamaño 8\% muestra \\
\hline 17 & 1 & 2 \\
20 & 2 & 4 \\
23 & 1 & 2 \\
44 & 2 & 4 \\
50 & 3 & 6 \\
66 & 3 & 6 \\
71 & 4 & 8 \\
353 & 18 & 24 \\
423 & 21 & 30 \\
\hline Total $=1067$ & Total $=55$ & Total $=86$ \\
\hline
\end{tabular}

Tabla 1. Tamaño de la muestra según conglomerados Fuente: Elaboración propia.

Por último, se redactó un Informe Científico Técnico también como parte de la formación en investigación, y un Informe de Divulgación para el Municipio y para la Junta Vecinal, a los efectos que pueda aportar para su gestión. 


\section{RESULTADOS, ANÁLISIS Y DISCUSIÓN}

\subsection{Resultados}

En primer lugar, se analizan tanto variables censales como variables perceptivas. Se obtiene datos referidos a edad, género, estado civil, lugar de nacimiento, tiempo de residencia en el Barrio, tiempo de residencia en Río Gallegos, tiempo en la vivienda, primer pariente radicado en Río Gallegos, estudios completos, ocupación, forma de tenencia de la vivienda, nombre del Barrio e identidad en relación a que piensa el encuestado cuando se le nombra Barrio Evita.

En lo referente a la edad, el mayor porcentaje (14\%) corresponde al grupo etario de 25 a 29 años; le siguen de 30 a 34 y de 40 a 44, es decir son personas jóvenes. Cabe aclarar que, de los adultos mayores, el porcentaje más alto corresponde al grupo etario 60 a 64 años. Respecto al género de los vecinos encuestados, el mayor porcentaje corresponde al género masculino, aunque es mínima la diferencia con el género femenino 51,25 y 48,8\% respectivamente femenino. (Gráfico 1)

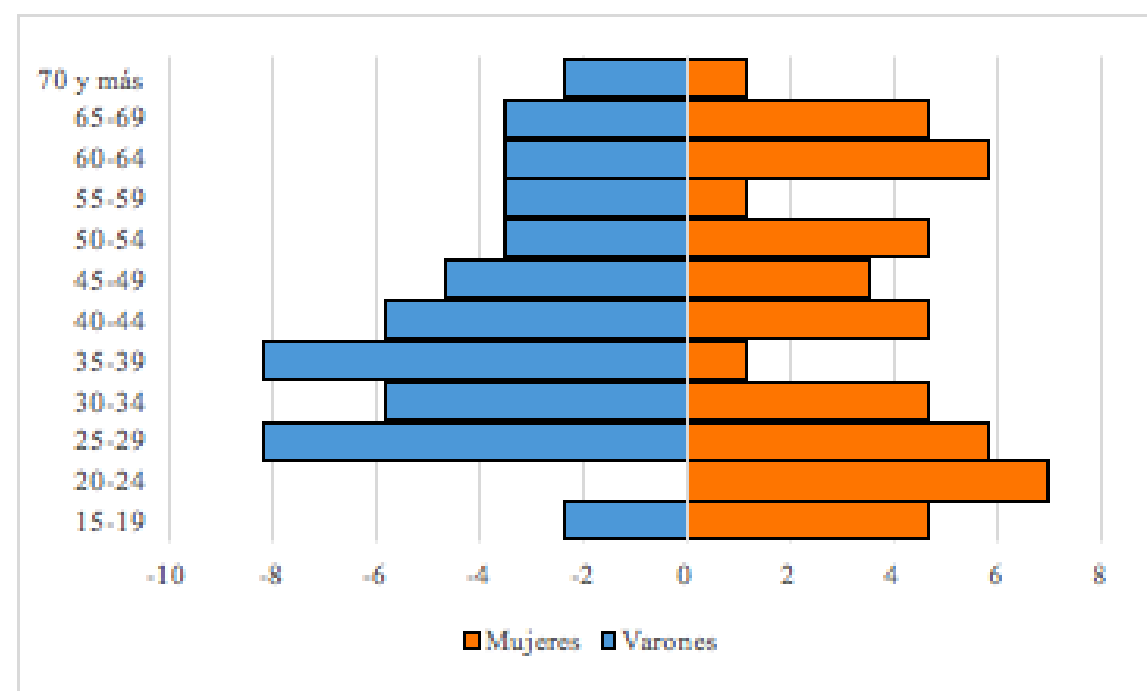

Gráfico 1. Población por edad y género, barrio Evita. Fuente: Elaboración propia.

Del estado civil de los encuestados, $51,2 \%$ son casados y el $37 \%$ son solteros. (Gráfico 2).

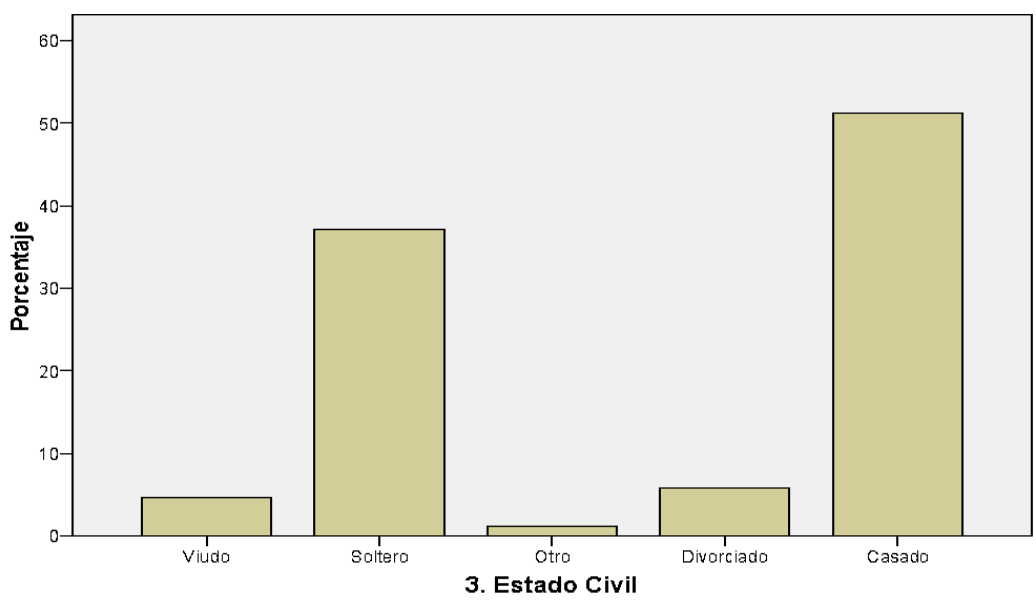

Gráfico 2. Estado civil de los habitantes del barrio Evita Fuente: Elaboración propia 
Con respecto al lugar de nacimiento el $73 \%$ son argentinos, de los cuales $88,8 \%$ son nacidos en Río Gallegos. De los extranjeros (26,7\%), el 100\% son nacidos en Chile, (Gráfico 3), cuyo lugar de procedencia es de la Patagonia Chilena: Punta Arenas $(47,8 \%)$, Puerto Natales $(30,43 \%)$ Puerto Montt (13\%), sólo el $8 \%$ son procedentes de Santiago de Chile. Los porcentajes de los nacidos en Río Gallegos son los mismos que en cuanto a procedencia, esto quiere decir que nacieron en la ciudad y no se fueron de la misma.

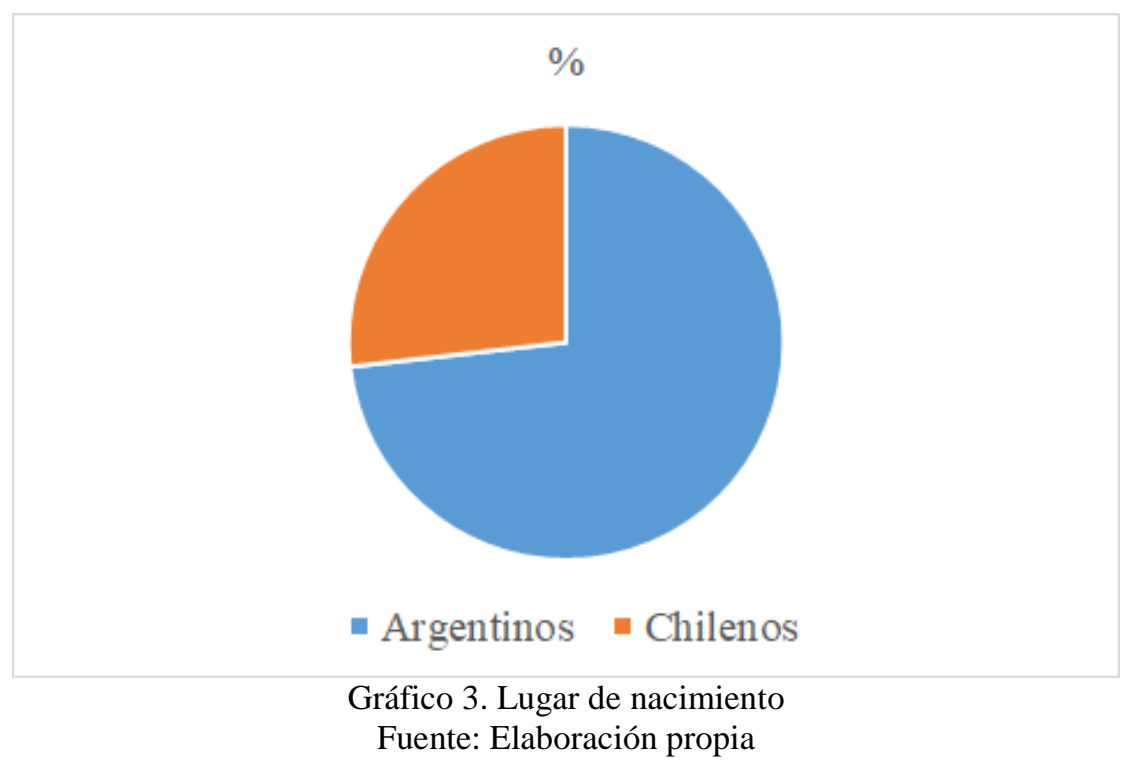

En cuanto al tiempo de residencia en el barrio Evita, se observa en primer lugar un grupo de personas con 1 a 14 años de residencia (26,7\%), le siguen de 15 a 19 años de residencia $(25,6 \%)$, de 20 a 24 años un 16,3\% y de 5 a 9 años un 18\%. (Tabla 2)

\begin{tabular}{cc}
\hline Años & Porcentaje \\
\hline $0-4$ & 5,8 \\
\hline $5-9$ & 18,6 \\
\hline $10-14$ & 26,7 \\
\hline $15-19$ & 25,6 \\
\hline $20-24$ & 16,3 \\
\hline $25-29$ & 3,5 \\
\hline $30-34$ & 1,2 \\
\hline
\end{tabular}

Tabla 2. Tiempo de Residencia en el Barrio Evita Fuente: Elaboración propia

Respecto al tiempo de residencia en Río Gallegos, se registró con un porcentaje de 17,4\% para aquellos que viven en la ciudad entre 20 a 24 años y 25 a 29 años, le sigue con un $12,8 \%$ de 15 a 19 años de residencia en la ciudad y un $11,6 \%$ de 35 a 39 años de residencia. (Tabla 3 ) 


\begin{tabular}{cc}
\hline Tiempo de residencia & Porcentaje \\
\hline $5-9$ & 2,3 \\
$10-14$ & 4,7 \\
$15-19$ & 12,8 \\
$20-24$ & 17,4 \\
$25-29$ & 17,4 \\
$30-34$ & 4,7 \\
$35-39$ & 11,6 \\
$40-44$ & 5,8 \\
$45-49$ & 7,0 \\
$50-54$ & 7,0 \\
$55-59$ & 1,2 \\
$60-64$ & 3,5 \\
$65-69$ & 1,2 \\
$70-74$ & 1,2 \\
$75-79$ & 1,2 \\
Fuente: Elaboración propia
\end{tabular}

Cuando se decide saber el primer pariente radicado en Río Gallegos del encuestado, es para conocer a qué número de generación en la ciudad pertenece, se adopta como criterio: encuestado primera generación, los padres segunda, los abuelos tercera y bisabuelos cuarta.

Los resultados detallados en la tabla 4 y representados en el gráfico 3 , dicen que predominan encuestados que pertenecen a la segunda generación.

Son que en lo referente al primer pariente del encuestado radicado en Río Gallegos y el número de generación radicada en la ciudad, se obtuvieron los siguientes resultados: el primer pariente radicado en la ciudad de Río Gallegos corresponde a un (43\%) por sus padres, teniendo en cuenta que sería la primera generación que se radica en la ciudad. (Tabla 4).

Se destaca que la primera generación radicada en la ciudad de Río Gallegos procede de la Patagonia Chilena (41,9\%). (Gráfico 3)

\begin{tabular}{cc}
\hline Pariente & Porcentaje \\
\hline Padres & 43,0 \\
No sabe & 24,4 \\
Encuestado/a & 1,2 \\
Bisabuelo/a & 4,7 \\
Abuelo/a & 23,3 \\
\hline
\end{tabular}

Tabla 4. Primer pariente radicado en Río Gallegos Fuente: Elaboración propia 


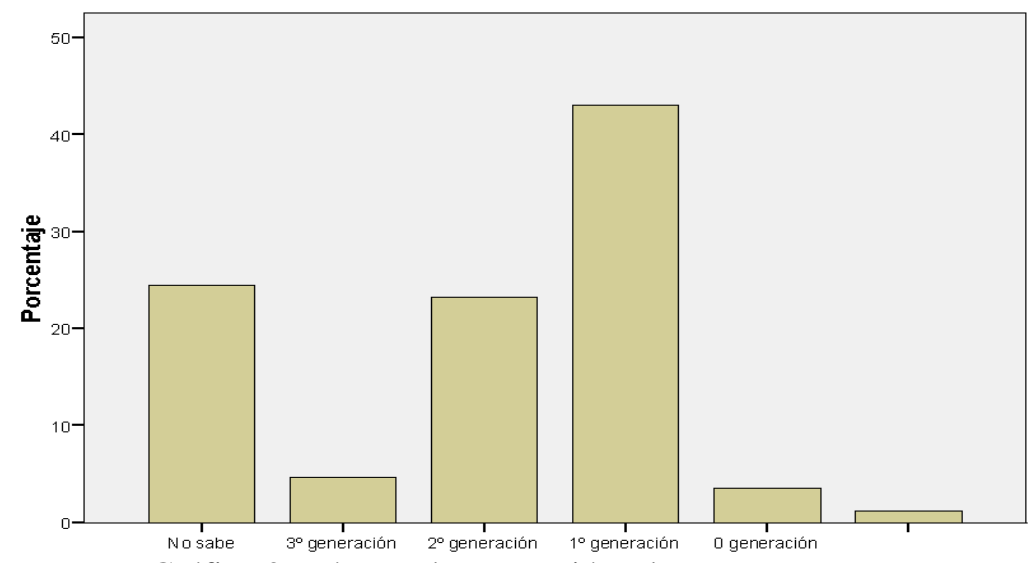

Gráfico 3. Número de generación a la que pertenece

Fuente: Elaboración propia

Respecto al nivel de estudios completados, un (59\%) tiene estudios secundarios completos, le siguen un $24,4 \%$ con primario completo y un $16,3 \%$ con un nivel superior completo. (Gráfico 4)

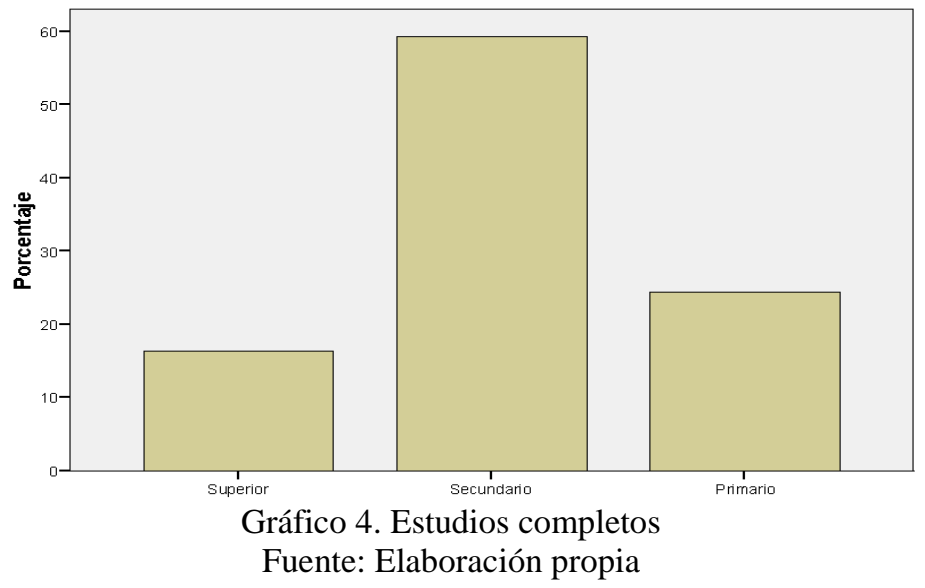

De la ocupación de los habitantes del barrio, se registró un predominio de empleados públicos provinciales (28\%). (Gráfico 5)

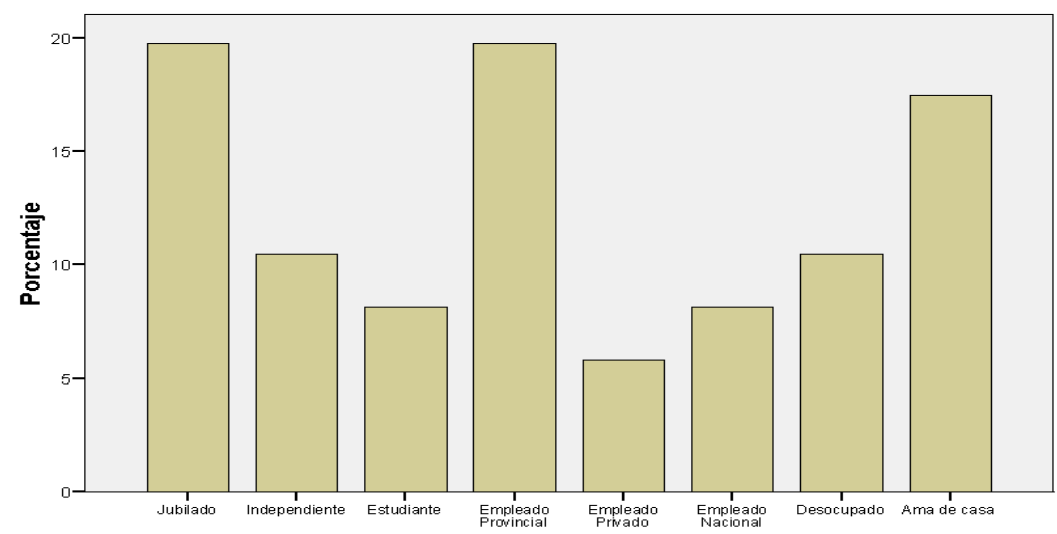

Gráfico 5. Ocupación

Fuente: Elaboración propia 
Respecto a la forma de tenencia de la vivienda, la mayor proporción de la población encuestada son propietarios $(65,1 \%)$. (Tabla 5).

\begin{tabular}{cc}
\hline Forma de tenencia & Porcentaje \\
\hline Propietario & 65,1 \\
Prestada & 17,4 \\
Inquilino & 17,4 \\
\hline
\end{tabular}

Tabla 5. Forma de tenencia de la vivienda Fuente: Elaboración propia

Del tiempo de residencia en la vivienda se registró un $15,1 \%$ de habitantes que viven hace 15 años en la vivienda. Le siguen las personas que viven hace 10 años en la vivienda (14\%) y 5 años en la vivienda (11,6\%). (Tabla 6)

\begin{tabular}{cc}
\hline Años & Porcentaje \\
\hline 1 & 1,2 \\
2 & 2,3 \\
3 & 2,3 \\
4 & 4,7 \\
5 & 14 \\
6 & 1,2 \\
7 & 1,2 \\
8 & 8,1 \\
9 & 1,2 \\
10 & 11,6 \\
11 & 3,5 \\
12 & 7 \\
13 & 1,2 \\
15 & 15,1 \\
17 & 2,3 \\
18 & 8,1 \\
20 & 9,3 \\
21 & 1,2 \\
22 & 1,2 \\
24 & 1,2 \\
25 & 1,2 \\
30 & 1,2 \\
\hline Total & 100 \\
\hline
\end{tabular}

Tabla 6. Tiempo de residencia en la vivienda Fuente: Elaboración propia 
Respecto al tiempo de residencia en la vivienda, se registró de 5 a 9 y de 15 a 19 años con un $(25,6 \%)$ y de 10 a 14 años un $(23,3 \%)$. (Tabla 7$)$

\begin{tabular}{cc}
\hline Años & Porcentaje \\
\hline $0-4$ & 10,5 \\
$5-9$ & 25,6 \\
$10-14$ & 23,3 \\
$15-19$ & 25,6 \\
$20-24$ & 12,8 \\
$25-29$ & 1,2 \\
$30-34$ & 1,2 \\
\hline Total & 100 \\
\hline \multicolumn{2}{c}{ Tabla 7. Tiempo en la vivienda } \\
Fuente: Elaboración propia
\end{tabular}

\subsection{Variables perceptivas}

Cuando se indagó sobre el nombre del barrio, un amplio porcentaje $(88,4 \%)$ de los encuestados reconocen el nombre Evita para el barrio. (Gráfico 6)

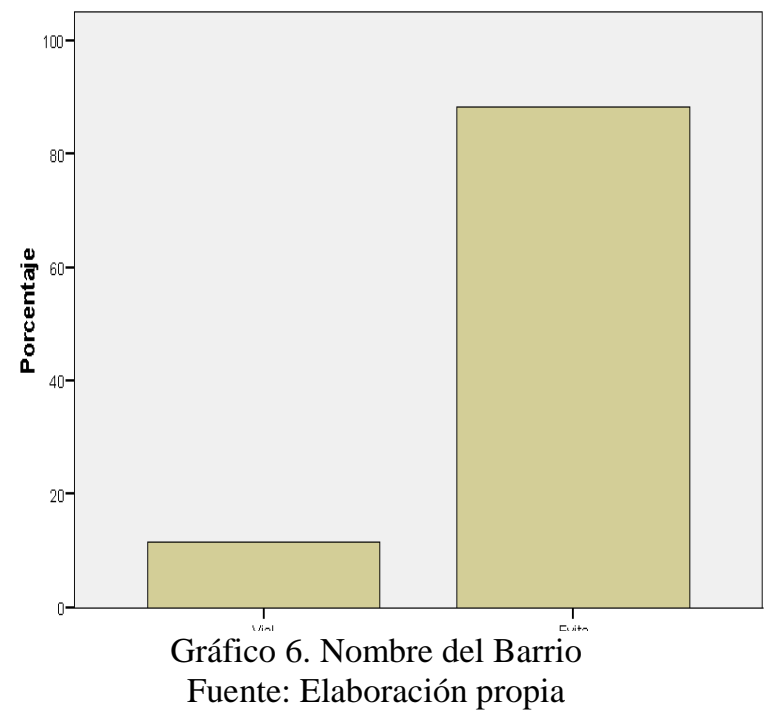

Respecto a los límites del barrio se reconocieron los siguientes (Fig. 4):

- Límite Norte: Zapiola $(90,7 \%)$

- Límite Oriental: Av. Balbín (80,2\%)

- Límite Occidental: Calle J. M de Rosas $(75,6 \%)$ 


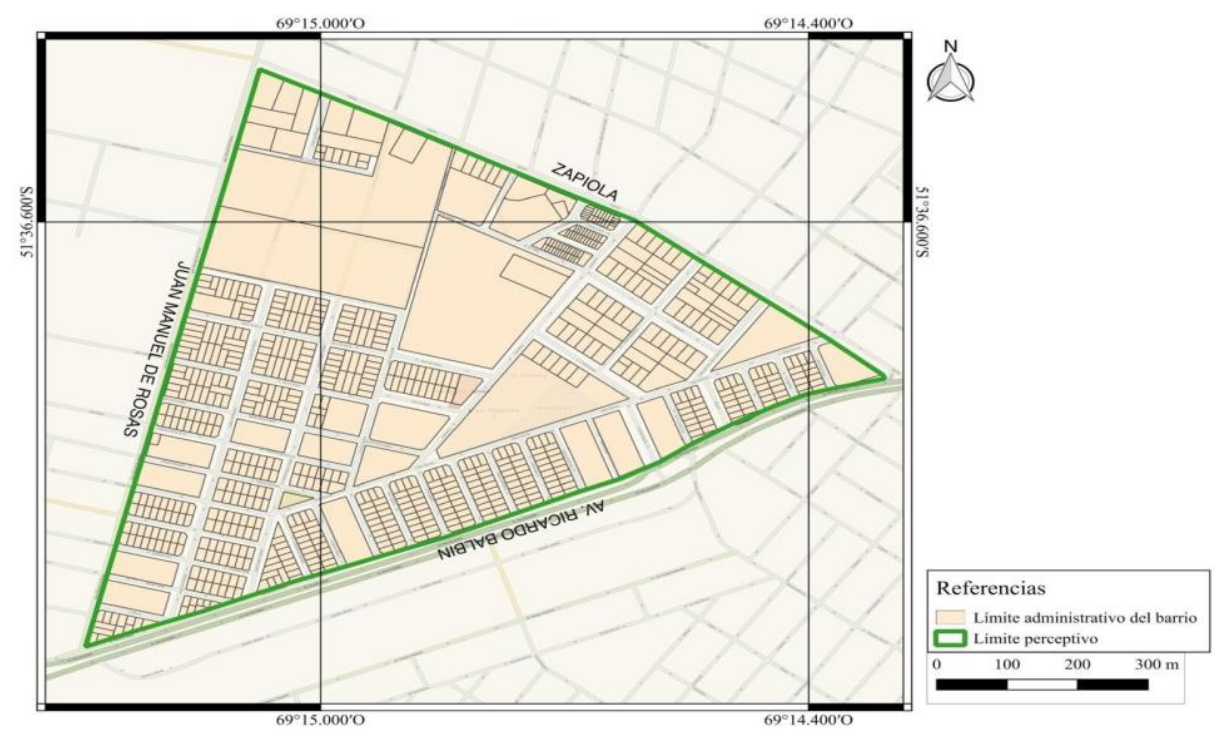

Fig. 4. Comparación de los límites administrativos con los perceptivos del barrio Evita Fuente: Elaboración propia en base a datos de la Municipalidad de Río Gallegos y encuestas realizadas en el barrio. Fecha: febrero de 2018.

Cabe aclarar que en porcentajes muy bajos las calles Pablo Neruda y Lola Mora también fueron percibidos como límites oriental y norte respectivamente.

Según los vecinos encuestados en el barrio Evita, lo primero que piensan cuando se le mencionó el barrio es, de forma decreciente: Inseguridad (30\%), calle Balbín (16,3\%), mi casa $(12,8 \%)$ y vías del tren $(10,5 \%)$. (Tabla 8$)$

\begin{tabular}{|c|c|}
\hline Pensamiento cuando se le menciona barrio Evita & Porcentaje \\
\hline Vecino & 1,2 \\
\hline Supermercado & 1,2 \\
\hline Perón & 1,2 \\
\hline Nada & 1,2 \\
\hline Lucho Fernández & 1,2 \\
\hline Lejanía & 1,2 \\
\hline Hipertehuelche & 1,2 \\
\hline Comunidad & 1,2 \\
\hline Comisaria & 1,2 \\
\hline Colegio & 1,2 \\
\hline Buena gente & 1,2 \\
\hline Basura & 1,2 \\
\hline Amistad & 1,2 \\
\hline Mi familia & 2,3 \\
\hline La Anónima & 2,3 \\
\hline Picadas de autos & 3,5 \\
\hline Perros sueltos & 3,5 \\
\hline Iglesia & 3,5 \\
\hline Vías del tren & 10,5 \\
\hline Mi casa & 12,8 \\
\hline Av. Balbín & 16,3 \\
\hline Inseguridad & 30,2 \\
\hline Total & 100 \\
\hline
\end{tabular}




\subsection{Discusión}

Del análisis de los resultados, se afirma que los límites perceptivos coinciden con los administrativos y los vecinos identifican claramente y están conformes con el nombre del barrio. Entre las variables censales analizadas se destacan las siguientes: nacidos en Río Gallegos (89\%) y de los extranjeros el $100 \%$ provienen de Chile; edad: predominan los jóvenes entre los 25 y 44 años; el tiempo de residencia en Río Gallegos con más de 20 años y en el barrio con menos de 20 años, con alto porcentaje en propietarios (65\%) como forma de tenencia; y en cuanto a la generación de nacidos y criados corresponden en su mayoría a la primera, debido que son los padres los primeros parientes que llegaron a Río Gallegos.

\section{CONCLUSIONES}

El problema planteado de una delimitación municipal del barrio Evita donde al barrio histórico se han incorporado otros sectores residenciales sin tener en cuenta la historia e identidad del mismo fue analizado y se concluye que a pesar de las diferencias entre sí en las características constructivas y socioeconómicas, los habitantes poseen una identidad barrial bien definida y homogénea para todo el sector estudiado. Por ello, el objetivo planteado de estudiar si la delimitación administrativa del barrio Evita de Río Gallegos coincide con los límites perceptivos que los habitantes poseen de su propio barrio, se ha cumplido, con la aplicación de la teoría y metodología del enfoque de la geografía de la percepción.

Los resultados de esta investigación, además de permitir a la becaria inciarse en el proceso de investigación en un equipo interdisciplinario, es de gran aporte tanto para el municipio como para la Junta Vecinal, para la gestión del barrio, porque demuestra cómo los vecinos perciben los límites del verdadero barrio.

\section{AGRADECIMIENTOS}

Se agradece al Lic. José Luis Sáenz por su valioso aporte en el desarrollo del muestreo estadístico, al Consejo Interuniversitario Nacional (CIN) por el financiamiento de la beca de investigación Estímulo a las Vocaciones Científicas (EVC) y a la UNPA por el financiamiento del Proyecto de Investigación de Desarrollo Tecnológico y Social (PDTS) 29/A380 "Tercera franja residencial de Río Gallegos: diagnóstico territorial de áreas con ausencia y presencia de cobertura de servicios. Propuesta de localización de nodos de servicios", en el cual se enmarcó la beca.

\section{REFERENCIAS}

AMPUERO, C. (2013): Percepción urbana de la localidad de El Calafate. Tesis de Licenciatura en Geografía. Universidad Nacional de la Patagonia Austral, Unidad Académica Río Gallegos. Inédita.

AMPUERO, C., CÁCERES, A. P. (2011): Accesibilidad geográfica de "nodos de servicios" en la segunda franja residencial de Río Gallegos, ciudad de la Patagonia austral Argentina. En Actas del XXXII Congreso Nacional y XVII Internacional de Geografía. Sociedad Chilena de Ciencias Geográficas. Valparaíso.

AMPUERO, C.; CÁCERES, A. P.; FRIAS, P., SÁENZ, J. L.; TRIVIÑO, G. (2014): Percepción de problemas urbanos de El Calafate, Santa Cruz, Argentina, en: Revista 
Contribuciones Científicas GÆA, n. 26. Sociedad Argentina de Estudios Geográficos. Buenos Aires.

ANDER-EGG, E. (1992): Técnicas en Investigación Social. Editorial Hvmanitas. Buenos Aires.

ARGENTINA. Instituto de Desarrollo Urbano y Vivienda (IDUV). Río Gallegos.

ARGENTINA. Instituto Nacional de Estadística y Censo (INDEC). Censos Nacionales 20012010. Buenos Aires.

CÁCERES, A. P.; SEGOVIA STANOSS, M.; SOTO, J.; NORAMBUENA, M.; FRIAS, P.; AMPUERO, C. (2016): Posición geográfica de Río Gallegos en la Patagonia Austral. Actas científicas del Congreso Internacional de Geografía - $77^{\circ}$ Semana de la Geografía. GÆA, Sociedad Argentina de Estudios Geográficos.

CÁCERES, A. P. (2013): Crecimiento urbano de la ciudad de Río Gallegos, capital de la provincia de Santa Cruz, Patagonia austral argentina (1885 - 2010), en: GEOUSAL Revista Científica de Geografía, año VIII, n. 14, Facultad de Historia, Geografía y Turismo de la Universidad del Salvador. Disponible en:

http://www.usal.edu.ar/archivos/geousal/docs/crecimiento_urbano_rio_gallegos_18852010.pdf Buenos Aires, Argentina.

CÁCERES, A. P. et al. (1996): Percepción urbana de Río Gallegos. Anales $20^{\circ}$ Sociedad Argentina de Estudios Geográficos. GÆA. Buenos Aires.

CÁCERES, A. P. et al. (2013): Informe final PI 29/A 244 "Geografía de la Percepción en la provincia de Santa Cruz, 20 años después de su primer abordaje". Universidad Nacional de la Patagonia Austral, Unidad Académica Río Gallegos. Río Gallegos.

CÁCERES, A. P. y GARCÍA, A. N. (2004): Diferenciación Interna del Espacio Geográfico de Río Gallegos: Estudio Preliminar. Revista del Instituto de Investigaciones Geográficas de la Patagonia. IGEOPAT. Año III. No 3. UNPSJB. Comodoro Rivadavia.

CÁCERES, A. P. y SOTO, J. (1996): Invierno del '95 en Río Gallegos: percepción y comportamiento. Sociedad Argentina de Estudios Geográficos. GÆA. Buenos Aires.

CÁCERES, A. P.; NORAMBUENA, M.; AMPUERO, C.; TRIVIÑO, G.: (2013): Consolidación de la tercera franja residencial de Río Gallegos, Patagonia Argentina. Actas Congreso Internacional de Geografía - $74^{a}$ Semana de Geografía. Trevelin (Chubut). Contribuciones Científicas GÆA. Vol. 25.

CÁCERES, A. P; SÁENZ, J. L.; AMPUERO, C.; WEBER, L. y ENRIQUE, L. (2004): Aplicación de una técnica de muestreo estratificado para una ciudad intermedia. Río Gallegos. Patagonia Austral. VI Jornadas Patagónicas de Geografía. UNPSJB. Trelew.

FRIAS, P.; CÁCERES, A. P; RAUQUE, M. (2011): Barrio Belgrano de Río Gallegos: diferencias entre la perspectiva de la delimitación y denominación barrial impuesta por el municipio y la vinculada a la identidad de los habitantes. Ponencia VIII Jornadas Patagónicas de Geografía. UNPSJB. Comodoro Rivadavia. Chubut.

GALLASTEGUI VERA, J.; GALEA ALARCÓN, J. (2008): El Barrio como unidad operativa para el desarrollo local. Editorial Lumen-Hvmanitas. Buenos Aires.

TRIVIÑO, J. G.; CÁCERES, A. P. (2010): Análisis y caracterización sociodemográfica de asentamientos en la segunda franja residencial de la ciudad de Río Gallegos. El caso particular del asentamiento Madres a la Lucha. Informe Científico Técnico UNPA-322011. Río Gallegos.

TRIVIÑO, J. G.; CÁCERES, A. P.; AMPUERO, C. (2017). Delimitación perceptiva de un sector residencial de la ciudad de Río Gallegos. Informe final de Beca Estimulo a las Vocaciones Científicas CIN - UNPA. Río Gallegos. 


\section{ANEXO 1}

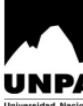

terra cognita

\begin{tabular}{|c|c|}
\hline Número de encuesta & \\
\hline UMTES PERCEPTIVOS BARRIOEVITA-BECA CIN UNPA
\end{tabular}

Fecha

Unaversidad Nacione

1. Edad
\begin{tabular}{|l|l|}
\hline 3. Estado Civil & Soltero \\
\cline { 2 - 2 } & Casado \\
\cline { 2 - 2 } & Viudo \\
\cline { 2 - 2 } & Divorciado \\
\cline { 2 - 2 } & Otro \\
\cline { 2 - 2 } & \\
\hline
\end{tabular}

\begin{tabular}{|l|l|l|l|l|l|l|l|}
\hline \multicolumn{7}{|c|}{ 6. Tiempo de Residencia en años } \\
\hline \multicolumn{3}{|c|}{ En el barrio o Sector } & \multicolumn{4}{|c|}{ En Río Gallegos } \\
\hline 1 & 10 & 20 & 30 & 1 & 10 & 20 & 30 \\
\hline
\end{tabular}

\section{Estudios completos alcanzados}

\begin{tabular}{|l|l|l|l|}
\hline Completos & Primario & Secundario & Superior \\
\hline
\end{tabular}

\section{8. ¿Quién fue el primer pariente que se radicó en la cuidad?}

\begin{tabular}{|l|l|l|l|l|}
\hline Parentesco & Año de radicación & Lugar de nacimiento & Procedencia (lugar en el cual vivió la mayor parte de su vida) \\
\hline
\end{tabular}

\begin{tabular}{|c|c|c|c|}
\hline & 9.00 & pación & \\
\hline & Ama de ca & & \\
\hline & Jubilado & & \\
\hline & Estudiant & & \\
\hline & Desocupac & & \\
\hline & Público & Nacional & \\
\hline Empleado & & Provincial & \\
\hline & Privado & & \\
\hline & dependier & & \\
\hline & Otros & & \\
\hline
\end{tabular}

10. Forma de Tenencia de la vivienda
\begin{tabular}{|l|l|}
\hline Es de su propiedad & \\
\hline Es alquilada & \\
\hline Es la vivienda familiar & \\
\hline Es prestada & \\
\hline Otra & \\
\hline
\end{tabular}

11. Tiempo de residencia en la vivienda

Localización

12. Domicilio:

13. ¿Cómo se llama este barrio?

14. Está conforme con el nombre que tiene? SI - NO

\footnotetext{
Límites del barrio

15. ¿Hasta dónde cree que son los límites del barrio? Limites 4 definidos

16. ¿Hasta dónde cree usted que deberían ser los limites el barrio?

17. ¿Qué es lo primero que se le viene a la mente cuando le menciono Barrio Evita?

18. ¿Qué calles utiliza para desplazarse en el barrio?

19. ¿Qué significa para Usted el barrio?
} 